\title{
Automatische Erkennung von epilepsietypischen Potenzialen und Anfällen im EEG
}

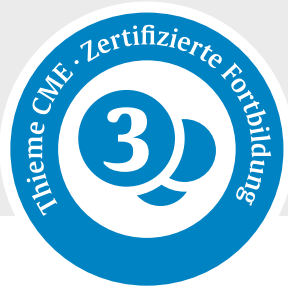

Die Elektroenzephalografie (EEG) ist der wichtigste apparative Eckpfeiler in der Diagnostik und Therapieführung bei Epilepsien. Die visuelle EEG-Befundung stellt dabei nach wie vor den Goldstandard dar. Automatische computerunterstützte Methoden zur Detektion und Quantifizierung von interiktalen epilepsietypischen Potenzialen und Anfällen unterstützen eine zeitsparende, objektive, rasch und jederzeit verfügbare quantitative EEG-Befundung.

\section{ABKÜRZUNGEN}

$\begin{array}{ll}\text { bPm } & \text { Beats per Minute } \\ \text { CNN } & \text { Convolutional Neural Network } \\ \text { EEG } & \text { Elektroenzephalografie } \\ \text { EMU } & \text { Epilepsie-Monitoring-Unit } \\ \text { ETPs } & \text { epilepsietypische Potenziale } \\ \text { FAR } & \text { false positive Alarm Rate } \\ \text { FIRDA } & \text { frontale intermittierende rhythmische } \\ & \text { Verlangsamung } \\ \text { FN } & \text { false negative } \\ \text { LSTM } & \text { Long Short Term Memory } \\ \text { RNN } & \text { Recurrent Neural Network } \\ \text { SUDEP } & \text { Sudden Unexpected Death in Epilepsy } \\ \text { TIRDA } & \text { temporale intermittierende rhythmische } \\ & \text { Verlangsamung } \\ \text { TP } & \text { true positive }\end{array}$

\section{FALLBEISPIEL}

\section{Fallbeispiel Frau E.: Anamnese und bisherige Untersuchungen}

\section{Anamnese}

Die 53-jährige Patientin leidet seit ihrem 13. Lebensjahr an Epilepsie.

\section{Anfallsbeschreibung:}

- Anfallstyp 1:

- Komisches, nicht näher zu beschreibendes Gefühl im Kopf, innere Unruhe, fühlt sich wie ferngesteuert, kann sprechen, versteht alles, kann sich an alles erinnern, Bewusstsein durchgehend erhalten.
- Frequenz: 3-4×/Monat.

- Anfallstyp 2:

- Entwickelt sich aus Anfallstyp 1, starrer Blick, keine Reaktion auf Ansprache, Bewusstseinsstörung, die Patientin erinnert diese Anfälle nicht.

- Frequenz: $2 \times /$ Monat.

- Anfallstyp 3:

- Entwickelt sich aus Anfallstyp 2, bilateraler tonisch-klonischer Anfall.

- Frequenz: 1-2×/Jahr.

Kein Status epilepticus in der Anamnese erhebbar. Schwangerschaft und Geburt: Zwillingsgeburt (Zweitgeborene), Saugglocke.

\section{Ätiologie:}

- rezidivierende Fieberkrämpfe im Alter von 1,5 Jahren,

- keine Meningitis oder Enzephalitis,

- kein signifikantes Schädel-Hirn-Trauma.

\section{Bisherige Untersuchungen}

- MRT-Schädel: mikroangiopathische Marklagergliosen, sonst unauffälliger Befund.

- Routine-EEG: gering abnorm, intermittierende Verlangsamung, generalisiert.

- Bisherige medikamentöse Therapieversuche: Carbamazepin, Lacosamid, Lamotrigin, Levetiracetam, Phenobarbital, Valproinsäure.

Die Patientin wird zur besseren Charakterisierung ihres Anfallsleidens zur Durchführung eines stationären intensiven Video-EEG-Monitorings in unsere Epilepsie-Monitoring-Unit aufgenommen. 


\section{Bedeutung der Elektroenzephalografie} in der Epileptologie

Die Erfassung von sog. epilepsietypischen Potenzialen

(ETPs) ist wichtig [1-2]

- in der Differenzialdiagnose von anfallsartigen Ereignissen,

- in der Prognoseabschätzung nach einem ersten Anfall,

- in der Klassifikation bzw. syndromatologischen Zuordnung von Epilepsien,

- in der Lokalisation der irritativen und Anfallsursprungszone bei fokalen Epilepsien (im Speziellen in der präoperativen Epilepsiediagnostik),

- in der Überprüfung der Therapiegüte bei generalisierten Epilepsien,

- in der Prognoseabschätzung bei fokalen Epilepsien,

- in der Diagnostik und Therapieführung beim Status epilepticus sowie

- in der neurologischen Intensivmedizin.

Obwohl die EEG-Aufzeichnung seit vielen Jahrzehnten digital erfolgt, stellt die visuelle Analyse der EEG-Rohdaten („EEG-Kurven“) nach wie vor den Goldstandard der EEGBefundung dar.

Merke

Das EEG ist nach wie vor die wichtigste apparative diagnostische Methode in der Epileptologie.

\section{Probleme bei der visuellen EEG-Befundung}

Bei der visuellen EEG-Befundung ergeben sich allerdings die folgenden Probleme [3-5]:

- Die visuelle EEG-Befundung ist zeitaufwendig (insbesondere die Analyse von Langzeitableitungen). Obwohl die EEG-Befundung durch erfahrene EEG-Befunder im Allgemeinen mit hoher Geschwindigkeit erfolgt, ergibt sich selbst bei 24-facher Analyse-Geschwindigkeit im Vergleich zur tatsächlichen Aufzeichnungszeit ein Zeitaufwand von einer Stunde zur Befundung einer 24-stündigen EEG-Aufzeichnung.

- Die visuelle EEG-Befundung ist anstrengend und ermüdend (insbesondere die Befundung von zahlreichen EEG-Ableitungen und die Analyse von Langzeitableitungen). Ermüdung und Ablenkung des EEG-Befunders sind also nicht auszuschließen, was die Befundqualität beeinträchtigen kann.

- Die EEG-Befundung erfordert eine spezielle Ausbildung und Erfahrung. Fehldiagnosen durch wenig erfahrene EEG-Befunder sind häufig.

- Auch unter qualifizierten und erfahrenden EEG-Befundern ist die Übereinstimmung bei der Annotation von ETPs keineswegs perfekt. Dies limitiert die Vergleich- barkeit der EEG-Befunde zwischen verschiedenen Einrichtungen, aber auch innerhalb einer Einrichtung.

- Die Verfügbarkeit von qualifizierten EEG-Befundern speziell außerhalb der Regelarbeitszeiten ist limitiert, weshalb wichtige klinische Entscheidungen oft erst verzögert getroffen werden können.

- Die klassische visuelle EEG-Beurteilung ermöglicht nur sehr eingeschränkt eine räumliche und zeitliche Quantifizierung von ETPs. Eine derartige Quantifizierung ist für die Prognoseabschätzung, die Therapieevaluation (bei generalisierten Epilepsien) und in der prächirurgischen Epilepsiediagnostik allerdings klinisch relevant.

FAZIT

Take Home Message

Die visuelle EEG-Befundung ist zeitaufwendig und ermöglicht speziell bei Langzeitableitungen nur sehr eingeschränkt eine quantitative Befunderstellung.

\section{Probleme bei der visuellen Annotation von interiktalen epilepsietypischen Potenzialen}

Aufgrund der Fortschritte in der computerunterstützenden Auswertung medizinischer Daten ist es deshalb naheliegend, diese Methoden auch auf die automatische computerunterstützte Detektion und Quantifizierung von ETPs anzuwenden, um so eine zeitsparende, objektive, reproduzierbare, rasch und jederzeit verfügbare, quantitative EEG-Befundung zu ermöglichen.

Interiktale epilepsietypische Potenziale sind definiert als EEG-Transienten, die sich klar von der Hintergrundaktivität abheben, eine negative Spitze aufweisen, von variabler Amplitude sind (im Idealfall $2 \times$ höher als die Hintergrundaktivität 5 s vor dem ETP) und eine spitze Morphologie aufweisen. Bei einer Dauer von 20-70 ms spricht man von einer Spitze (Spike), bei einer Dauer von 70-200 ms von einer scharfen Welle (Sharp-Wave) [6]. Trotz dieser scheinbar klaren Definition kommt es in der klinischen Praxis häufig vor, dass eine EEG-Transiente von einem erfahrenen EEG-Befunder als eindeutiges ETP bezeichnet wird, während eine morphologisch ähnliche - für einen unerfahrenen EEG-Neuling scheinbar identische - Transiente als nicht epilepsietypisch klassifiziert wird [5].

In diesem Zusammenhang muss auf die häufige Fehl- bzw. Überinterpretation von EEGs speziell durch unerfahrene EEG-Befunder hingewiesen werden. Hier sind einerseits Artefakte und andererseits sog. marginale EEG-Muster (benigne EEG-Muster mit epileptiformer Morphologie) wie z. B. Wicket-Spikes, benigne epileptiforme Transienten des 
Schlafs, $14 \& 6 \mathrm{~Hz}$-positive Spikes, Phantom-Spike-WaveKomplexe, ein Breach-Rhythmus u. Ä. zu nennen [7, 8]. Falsch-negative Befunde können zur Verzögerung oder zum Vorenthalt einer effektiven Therapie führen, falschpositive Befunde hingegen zu Fehldiagnosen und langdauernden Fehlbehandlungen mit entsprechenden nachteiligen Folgen für die betroffenen Patienten [8].

FAZIT

\section{Take Home Message}

Die Fehlbefundung eines EEGs kann zu Fehldiagnosen und mitunter langjährigen Fehlbehandlungen führen.

Andererseits können auch bei qualifizierten und erfahrenen EEG-Befundern beträchtliche interindividuelle Diskrepanzen in der Zuordnung von ETPs bestehen [9-11]. Die Übereinstimmung von zertifizieren EEG-Befundern in akademischen Institutionen mit einer EEG-Spezialausbildung ist dabei größer als die von niedergelassenen EEG-Befundern ohne Zusatzqualifikation [9, 10].

In einer rezenten Studie [4] zeigte sich zwar eine substanzielle Übereinstimmung von 9 erfahrenen EEG-Befundern hinsichtlich des Vorhandenseins von ETPs in einem gegebenen EEG (mittlere prozentuale Übereinstimmung $80,9 \%$, mittleres Kappa 69,4\%), die Übereinstimmung bei der Annotation einzelner ETPs war jedoch lediglich moderat (mittlere prozentuale Übereinstimmung 72,4\%, mittleres Kappa 48,7\%). Die Autoren erklären die Unterschiede bei der Annotation einzelner ETPs durch unterschiedliche subjektive individuelle Schwellenwerte der verschiedenen EEG-Befunder für die binäre Entscheidung ETP vs. kein ETP, die sich durch wenige morphologische Eigenschaften erklären ließen [4].

Cave

Bei der visuellen EEG-Befundung besteht auch in erfahrenen Händen eine beträchtliche interindividuelle Variabilität.

\section{Qualitätskriterien bei der}

automatischen Detektion von

interiktalen epilepsietypischen

\section{Potenzialen}

Die Qualität eines Algorithmus zur Detektion von interiktalen ETPs wird an dessen Sensitivität und Spezifität gemessen.

\section{Sensitivität}

Die Sensitivität ist ein Maß für die Erfassung von ETPs. Algorithmen mit hoher Sensitivität detektieren nahezu alle
ETPs, bei niedriger Sensitivität können relevante ETPs hingegen unerkannt bleiben.

\section{Spezifität}

Die Spezifität reflektiert die Zahl der falsch-positiven Detektionen. Bei einer hohen Spezifität ist die Zahl der falsch-positiven Detektionen niedrig, bei niedriger Spezifität hingegen gibt es eine große Zahl von falsch-positiven Detektionen (bedingt durch Artefakte oder nicht-epilepsietypische Transienten).

Die Konsequenz einer niedrigen Spezifität für die klinische Praxis ist, dass - aufgrund der hohen Zahl der Detektionen letztlich ein Großteil des EEGs visuell reanalysiert werden muss. Dies ist dann letztlich zeitaufwendiger als die traditionelle visuelle Analyse per se, womit eine derartige automatische Detektion nutzlos wird. Viele Algorithmen verwenden deshalb ein mehrstufiges Vorgehen, bei dem zunächst möglichst viele scharfe Transienten detektiert werden und dann in einem zweiten Schritt Artefakte und nicht-epilepsietypische Transienten gezielt verworfen werden.

Das Problem der meisten Algorithmen besteht darin, dass eine hohe Sensitivität nur auf Kosten einer hohen Rate von falschen Alarmen erreicht werden kann und umgekehrt eine niedrige Rate von falschen Alarmen nur auf Kosten einer reduzierten Sensitivität. In der Literatur werden für automatische Spike-Detektionsprogramme durchschnittliche Sensitivitäten zwischen 65 und $99 \%$ sowie durchschnittliche falsch-positive Detektionen zwischen 0,09 und 13,4 pro Minute berichtet [12].

\section{FAZIT}

\section{Take Home Message}

Automatische Spike-Detektionsprogramme verfügen über Sensitivitäten von 65-99\%, bei 0,09-13,4 falsch-positiven Detektionen pro Minute.

\section{Goldstandard für die automatische Detektion von interiktalen epilepsietypischen Potenzialen}

Ein weiteres Problem bei der Überprüfung der Qualität eines Spike-Detektionsprogramms besteht in der Definition eines geeigneten Goldstandards.

\footnotetext{
Merke

Der Goldstandard ist dabei nach wie vor die visuelle Annotation von ETPs durch qualifizierte und erfahrene EEG-Befunder.
}

Allerdings müssen hierbei die bereits oben angeführten Diskrepanzen bei der visuellen Annotation von ETPs durch unterschiedliche EEG-Befunder berücksichtigt werden. 
Welcher EEG-Befunder definiert also den Goldstandard? Eine Möglichkeit besteht darin, den Goldstandard als Konsens verschiedener EEG-Befunder festzulegen. Allerdings bildet bei dieser Vorgehensweise ein automatisches SpikeDetektionsprogramm die klinische Befundroutine keines einzigen individuellen EEG-Befunders mehr ideal ab, d. h. individuelle EEG-Befunder werden immer Diskrepanzen zwischen dem Spike-Detektionsprogramm und sich selbst finden.

Alternativ könnte man das Spike-Detektionsprogramm auf einzelne EEG-Befunder individuell trainieren und zuschneiden. Dieses Vorgehen wäre allerdings mit einem beträchtlichen Zeitaufwand verbunden, zudem wären die Ergebnisse von derartig individualisierten Spike-Detektionsprogrammen bei ein und demselben Patienten unterschiedlich, und die Ergebnisse zwischen und möglicherweise sogar innerhalb von klinischen Einrichtungen nicht mehr vergleichbar [5].

Ein möglicher Ausweg aus diesem Dilemma ist es, SpikeDetektionsprogramme nicht mit einem Goldstandard, sondern mit mehreren erfahrenen EEG-Befundern zu vergleichen, also einen statistischen Turing-Test anzuwenden. Die Frage ist dann, ob die Unterschiede zwischen den menschlichen EEG-Befundern größer oder kleiner sind als die zwischen menschlichen EEG-Befundern und dem computerbasierten Spike-Detektionsprogramm [11].

Tatsächlich konnte das kommerziell verfügbare Spike-Detektionsprogramm Persyst 13 eine vergleichbare Sensitivität und Spezifität wie 3 erfahrene EEG-Befunder erzielen [11]. Die Sensitivitäten und korrespondierenden falschen Alarmraten pro Minute für die menschlichen EEG-Befunder waren 40,0, 42,1 und 51,5\% bzw. 0,80, 0,97 und 1,99/ min, für den Persyst 13 Algorithmus 43,9\% bzw. 1,65/min.

In einer rezenten Studie zeigte das Spike-Detektionsprogramm SpikeNet eine bessere Performance als 8 erfahrene EEG-Befunder sowohl hinsichtlich der Detektion individueller ETPs als auch hinsichtlich der Klassifikation einer gesamten EEG-Ableitung bezüglich des Vorhandenseins bzw. Fehlens von ETPs [13].

\section{FAZIT}

Take Home Message

Automatische Spike-Detektionsprogramme erbringen eine vergleichbare oder sogar bessere Performance als erfahrene menschliche EEG-Befunder.
Spike-Merkmale bei der SpikeAnnotation durch menschliche EEG-Befunder

Nachdem bei der Spike-Annotation die Übereinstimmung verschiedener EEG-Experten nicht perfekt ist, ist es für die Entwicklung eines automatischen Spike-Detektionssystems durchaus relevant festzustellen, welche Spike-Merkmale zur Übereinstimmung bei der Spike-Annotation von menschlichen EEG-Experten entscheidend beitragen. In einer Studie, in der 18 klinische Neurophysiologen SpikeAnnotationen in 200 EEG-Segmenten von 30 s Dauer bei 200 Patienten durchführten, konnten bestimmte WaveletMerkmale die Übereinstimmung der EEG-Experten am besten vorhersagen. Diese Eigenschaften können wiederum als Basis von Machine-Learning-Algorithmen genutzt werden [3].

\section{Methoden der automatischen Spike-Detektion}

Die Umstellung von der analogen auf die digitale EEGTechnologie bedeutete einen Anstoß für die Entwicklung von automatischen computergestützten Spike-Detektionsverfahren in den frühen 1970 er-Jahren. Die Methoden der automatischen Spike-Detektion wurde in mehreren exzellenten Review-Artikeln umfassend dargestellt [1417]. Zu nennen sind dabei u. a. sog. Template-MatchingMethoden, parametrische (autoregressive) Methoden, mimetische Methoden, frequenzanalytische Methoden bzw. die Power-Spektrum-Analyse und die Wavelet-Analyse. Zudem kamen auch die Unabhängigkeitsanalyse (Independent Component Analysis), die Dipolquellenanalyse (Electrical Source Imaging) und Methoden des maschinellen Lernens (Machine Learning) für die automatische SpikeDetektion zur Anwendung.

Eine neue Entwicklung ist die Anwendung von sog. DeepLearning-Methoden (mehrschichtiges Lernen, tiefes Lernen oder tiefgehendes Lernen) für die automatische SpikeDetektion [18]. In einer rezenten Studie zur Spike-Detektion mittels Deep Learning kamen 2 Netzwerktypen zur Anwendung, nämlich Convolutional Neural Networks (CNNs) mit Feedforward-Konnektivität und Long Short Term Memory (LSTM)-Netzwerke, ein spezieller Typ von Recurrent Neural Networks (RNNs) [18]. Zum Training des Netzwerks wurden 41,381 EEG-Segmente (Dauer jeweils 2 s) mit 1478 ETPs verwendet. Die Evaluation erfolgte dann an einem anderen, vergleichbaren Datensatz mit 8,775 EEG-Segmenten (Dauer erneut jeweils 2 s) mit 337 ETPs. Dabei konnte eine Sensitivität von $47,4 \%$ und eine Spezifität von 98,0\% (0,6 falsch-positive Detektionen pro Minute) erreicht werden, bei normalen EEGs betrug die Häufigkeit von falsch-positiven Detektionen 0,3 pro Minute [18]. Damit war die Sensitivität deutlich niedriger $[12,17,19]$ 
bzw. vergleichbar $[11,12,17]$ wie bei klassischen manuell adjustierbaren Spike-Detektionsprogrammen.

Der Vorteil des Deep-Learning-Ansatzes besteht allerdings darin, dass keine subjektiven, manuellen Adjustierungen erforderlich sind und dass die eher niedrige Sensitivität durch weiteres Training an größeren Datenmengen, Erhöhung der Netzwerkgröße, Optimierung der Netzwerkarchitektur und geringe Reduktion der sehr hohen Spezifität noch deutlich erhöht werden könnte [20]. Die höhere Rate von falsch-positiven Detektionen bei Patienten mit fokalen Epilepsien als bei normalen Kontrollen könnte auch ein Hinweis darauf sein, dass Deep-Learning-Algorithmen Merkmale im EEG erkennen, die der visuellen Analyse nicht zugänglich sind [21].

\section{Merke}

Deep-Learning stellt eine vielsprechende Entwicklung in der automatischen EEG-Befundung dar.

In $>$ Abb. 1 und $\mathbf{2}$ sind die Ergebnisse einer automatischen computerunterstützten Spike-Detektion und einer automatischen Analyse der räumlich-zeitlichen Spike-Verteilung mithilfe des Spike-Detektions-Moduls EpiSpike des EEG-Auswerteprogramms Encevis bei einer Patientin mit rechtshirniger Temporallappenepilepsie dargestellt.

\section{FALLBEISPIEL}

\section{Fallbeispiel Frau E.: Video-EEG-Monitoring - inter- iktales EEG}

Die visuelle EEG-Beurteilung ergibt den Befund „häufige“ Spikes rechts temporal. Zudem kam eine automatische computerunterstützte Spike-Detektion und räumlich-zeitliche Spike-Analyse mit dem Spike-Detektions-Modul EpiSpike des Auswerteprogramms Encevis zur Anwendung.

- Abb. 1 zeigt einen durch das Programm detektierten Spike rechts temporal (Maximum an der Elektrode FT10).

In $>$ Abb. $\mathbf{2}$ sind die Ergebnisse der Auswertung des automatischen Spike-Detektions- und Analyseprogramms zusammengefasst:

Während der gesamten Ableitdauer von 4 Tagen wurden insgesamt 432 Spikes detektiert, alle über der rechten Hemisphäre (d. in > Abb. 2).

Die beiden häufigsten Spike-Typen bzw. SpikeCluster zeigten ein Maximum an der Elektrode F8 (204 Spikes [47,2\% aller Spikes]) bzw. an der Elektrode FT10 (160 Spikes [37,0\% aller Spikes])

(a. in $\mathbf{A b b}$. 2).

Das (gemittelte) EEG von individuellen Spike-Clustern ist in $\mathbf{b}$. in > Abb. $\mathbf{2}$ visuell dargestellt (hier für den Spike-Cluster mit Amplitudenmaximum an der Elektrode FT10).

c. in $>$ Abb. 2 zeigt das Auftreten von einzelnen Spikes innerhalb der verschiedenen Spike-Cluster im zeitlichen Verlauf. Bei unserer Patientin zeigt sich eine deutliche zeitliche Häufung der Spikes an den Elektroden F8 und FT10 im Zeitraum zwischen 00:00 und 00:30.

Die automatische computerunterstützte SpikeDetektion und Spike-Auswertung ermöglichen somit eine detaillierte Analyse der räumlichen und zeitlichen Spike-Verteilung.

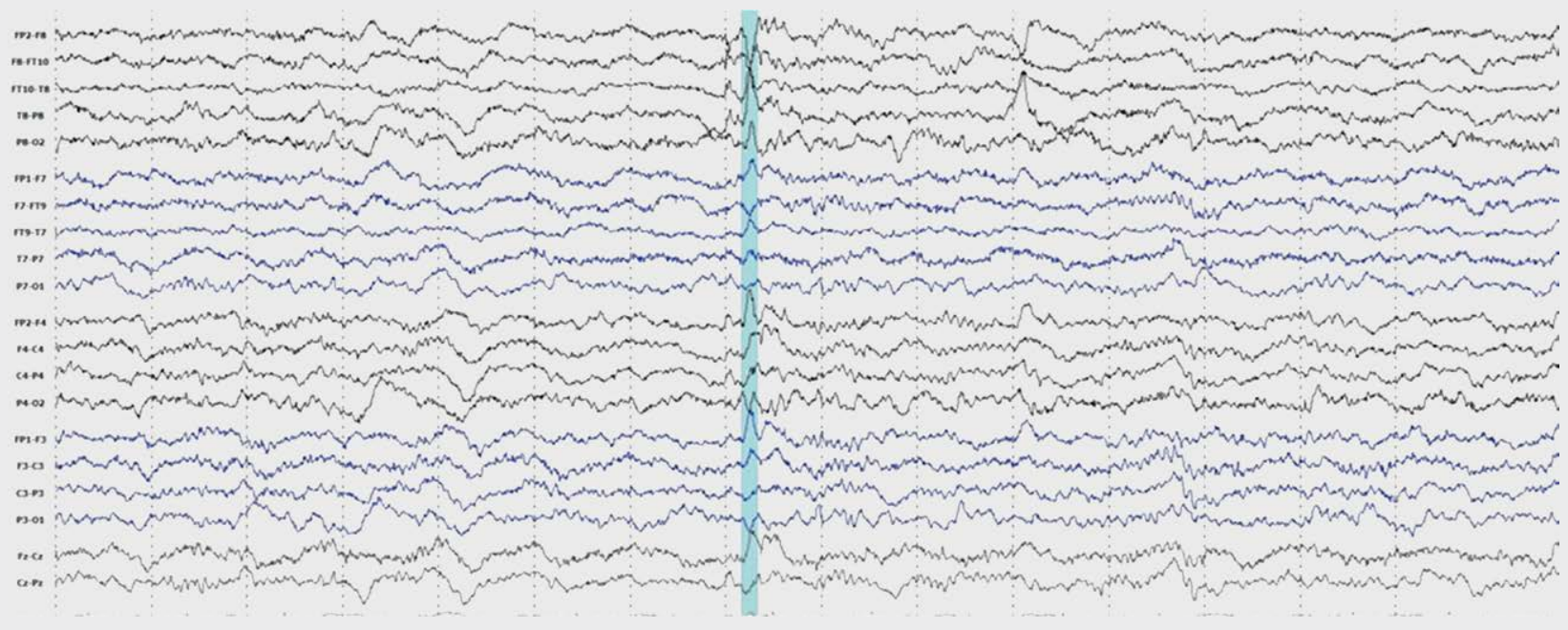

- Abb. 1 Interiktales EEG - Automatische Spike-Detektion. Durch das Spike-Detektions-Modul EpiSpike des Auswerteprogramms Encevis detektierter Spike rechts temporal (Maximum an der Elektrode FT10 - hellblau hinterlegt). 

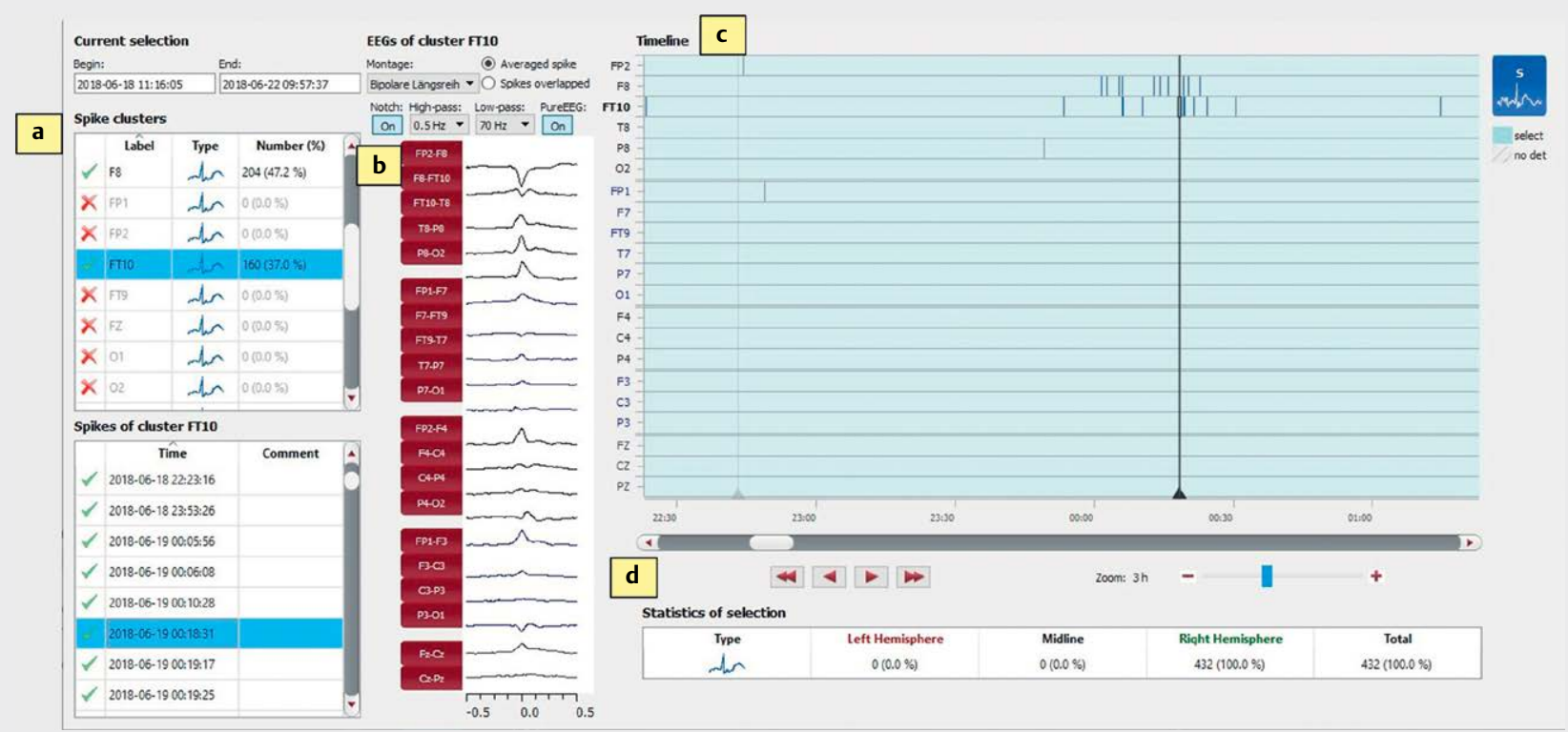

Abb. 2 Automatische Spike-Detektion und räumliche-zeitliche Analyse der Spike-Cluster mit dem Spike-Detektionsmodul EpiSpike des Auswerteprogramms Encevis. a. Relative Häufigkeit der einzelnen Spike-Cluster. b. Visuelle Darstellung des (gemittelten) EEGs von individuellen Spike-Clustern. c. Darstellung des Auftretens von Spikes der verschiedenen Spike-Cluster im zeitlichen Verlauf. d. Zusammenfassende Statistik der Spike-Detektionen über der rechten und der linken Hemisphäre.

\section{Das Anfallserfassungsproblem}

Anfallsfrequenz und Anfallsschwere sind die wesentlichen Zielparameter sowohl in der täglichen klinischen Epilepsiebehandlung als auch in der Beurteilung der Effektivität von medikamentösen und nicht-medikamentösen therapeutischen Interventionen im Rahmen klinischer Studien. Allerdings ist die von den betroffenen Patienten und ihren Angehörigen durchgeführte Anfallsdokumentation äußerst unzuverlässig. So wurden in einer Studie an einem hochselektionierten Patientenklientel mit fokalen Epilepsien während des stationären Video-EEG-Monitorings mehr als $50 \%$ aller Anfälle, $73 \%$ der fokalen, nicht bewusst erlebten Anfälle und $86 \%$ der Anfälle aus dem Schlaf von den Patienten selbst nicht bemerkt [22]. Dieses Problem wird als sog. Anfallserfassungsproblem bezeichnet und stellt eines der zentralen Probleme der klinischen Epileptologie dar [23].

\section{Merke}

Das Anfallserfassungsproblem besteht darin, dass die Mehrzahl der fokalen, nicht bewusst erlebten Anfälle und der Anfälle aus dem Schlaf von den betroffenen Patienten nicht bemerkt werden.

Es besteht deshalb ein dringender klinischer Bedarf für automatische computerbasierte Anfallsdetektionssysteme zur objektiven Anfallsdokumentation, wodurch ein wichtiger Beitrag zur Lösung des Anfallserfassungsproblems geleistet werden könnte [24-26]. Derartige Systeme könnten auch als Anfallswarnsysteme eingesetzt werden, wodurch die Unvorhersehbarkeit der Anfälle und deren soziale Konsequenzen abgemildert, anfallsassoziierte Verletzungen verhindert und ein Beitrag zur Prävention von Sudden Unexpected Death in Epilepsy (SUDEP) geleistet werden könnte [27]. Automatische Anfallsdetektionssysteme sind schließlich eine entscheidende Komponente für innovative bedarfsgesteuerte Therapien, wie die anfallsgetriggerte lokale Freisetzung von Antikonvulsiva oder elektrische Stimulation, um so Anfälle zu unterbrechen oder gar nicht entstehen zu lassen [23].

\section{Automatische Anfallsdetektion in der Epilepsie-Monitoring-Unit}

Wichtige Anwendungsmöglichkeiten für automatische Anfallsdetektionssysteme im Rahmen des stationären VideoEEG-Monitorings in der Epilepsie-Monitoring-Unit bestehen in

- der Optimierung der Patientensicherheit,

- der Ökonomisierung der Datenauswertung mit objektiver und reproduzierbarer Quantifizierung von Anfallsmustern und

- der automatisierten computergestützten neurologischen bzw. neuropsychologischen iktalen und postiktalen Patiententestung.

Im Fokus der Patientensicherheit in der Epilepsie-Monitoring-Unit stehen die rechtzeitige Erkennung und Behandlung von Anfällen, die Verhinderung des Auftretens von Status epileptici, die Vermeidung von anfallsassoziierten Verletzungen, die SUDEP-Prävention und schließlich die unvermittelte Erkennung und allfällige Behandlung von anfallsassoziierten kardialen Rhythmusstörungen [28]. 
Merke

Eine maximal mögliche Patientensicherheit kann grundsätzlich nur durch kontinuierliche Überwachung durch qualifiziertes Personal gewährleistet werden [28, 29], die allerdings nur in 56-80\% der EMUs vorgehalten werden kann [28]. Automatische Anfallsdetektions- bzw. Anfallsalarmsysteme könnten in diesen Situationen die Patientensicherheit signifikant verbessern.

Automatische Anfallsdetektionssysteme können auch die zeitaufwendige EEG-Befundung signifikant optimieren [30]. Die Analyse der klinischen Anfallssemiologie erbringt entscheidende Hinweise auf Anfallsursprung und -propagation, wobei eine systematische interaktive Testung der Patienten während und nach dem Anfall hier entscheidende Zusatzinformation erbringt [31]. Allerdings kann eine solche Testung unter klinischen Routinebedingungen oft nicht oder nur verzögert durchgeführt werden. Eine automatisierte, computergestützte, durch ein EEG-Anfallsdetektionssystem getriggerte Anfallstestung könnte hier Abhilfe schaffen [32].

FAZIT

Take Home Message

Während des stationären Video-EEG-Monitorings könnten durch automatische Anfallsdetektionssysteme die Patientensicherheit verbessert, die Datenauswertung beschleunigt und objektiviert sowie die Patiententestung automatisiert werden.

\section{Anforderungen an automatische Anfallsdetektionssysteme}

Die visuelle EEG-Analyse durch erfahrene und qualifizierte EEGExperten stellt den Goldstandard für die Anfallserkennung und -bewertung dar. Generell ist die Übereinstimmung zwischen verschiedenen EEG-Experten bei der Identifikation von EEGAnfallsmustern hoch (durchschnittliche übereinstimmende Sensitivität $92 \%$ und 0,117 falsch-positive Identifikationen pro Stunde). Dennoch können eine hohe Anfallsfrequenz (mehrere Anfälle pro Stunde), eine kurze ( $<10$ s) sowie eine lange Anfallsdauer ( $10 \mathrm{~min}$ ) mit unklarem EEG-Anfallsende die visuelle Analyse mühsam machen und die Übereinstimmung zwischen unterschiedlichen EEG-Befundern beeinträchtigen [33]. Automatische Anfallsdetektionssysteme könnten speziell in diesen Situationen eine objektive und reproduzierbare Quantifizierung von Anfallsmustern erleichtern.

\section{Merke}

Ein automatisches Anfallsdetektionssystem muss unterschiedliche Anfallstypen bei unterschiedlichen Patienten mit unterschiedlichen Epilepsiesyndromen mit hoher Sensitivität und hoher Spezifität detektieren.

\section{Sensitivität}

Die Sensitivität ist dabei definiert als Quotient von TP/ $(\mathrm{TP}+\mathrm{FN})$.

- TP bedeutet true positives oder richtig-positive, d. h. Anfälle, die sowohl vom EEG-Experten als auch vom Anfallsdetektionssystem als Anfälle erkannt werden.

- FN bedeutet false negatives (FN) oder falsch-negative, d. h. Anfälle, die vom EEG-Experten erkannt werden, nicht aber vom Anfallsdetektionssystem.

\section{Spezifität}

Die Spezifität wird definiert über die Anzahl von falsch-positiven Alarmen (false positive Alarm Rate, FAR) pro Zeiteinheit [24-26, 34]. Die Anforderungen an die Spezifität hängen auch von der Anwendung ab. So können bei einer automatischen Anfallsdetektion in der Epilepsie-Monitoring-Unit falsch-positive Alarme eher hingenommen werden als in Patientenwarnsystemen.

\section{Einsatz als Alarmsystem}

Ein Anfallsdetektionssystem ist nur dann als Alarmsystem brauchbar, wenn eine Online-Detektion mit kurzer Detektionslatenz von wenigen Sekunden möglich ist. Die Detektionslatenz ist dabei definiert als die zeitliche Verzögerung zwischen dem vom EEG-Experten visuell festgelegten Anfallsbeginn im EEG und dem Zeitpunkt, zu dem der Alarm durch das Detektionsprogramm gesetzt wird [35].

\section{FAZIT}

Take Home Message

Ein automatisches Anfallsdetektionssystem soll Anfälle mit hoher Sensitivität bei niedriger falsch-positiver Alarmrate detektieren. Bei Alarmsystemen ist zudem eine Online-Detektion mit kurzer Detektionslatenz erforderlich.

\section{FALLBEISPIEL}

Fallbeispiel Frau E.: Video-EEG-Monitoring - Anfälle und iktales EEG

Während des Monitorings konnten 2 habituelle Anfälle abgeleitet werden. Die Patientin verspürte dabei (wie schon anamnestisch berichtet) ein komisches, nicht näher beschreibbares Gefühl im Kopf, dann eine innere Unruhe, nachfolgend dann Innehalten, die Patientin starrte ins Leere, reagierte nicht auf Ansprache, postiktal war bei einem Anfall ein repetitives Wassertrinken zu beobachten. Die klinische Anfallssemiologie deutet somit auf einen Anfall aus dem rechten Temporallappen hin (postiktales Wassertrinken als lateralisierendes Zeichen für einen Anfallsursprung in der nicht- 
sprachdominanten Hemisphäre).

Die visuelle EEG-Beurteilung ergibt den Befund eines rechtstemporalen Anfallsmusters (rhythmische Theta-Tätigkeit).

Zudem erfolgten eine automatische Anfallsdetektion mit dem Anfalls-Detektions-Modul EpiScan und eine automatische Analyse des EEG-Verlaufs mit dem EEG-Trend-Modul NeuroTrend des EEG-Auswerteprogramms Encevis über die gesamte Ableitdauer.

Abb. 3 zeigt ein durch das Anfalls-DetektionsModul EpiScan detektiertes rechtstemporales Anfallsmuster.

\section{Algorithmen zur automatischen Anfallsdetektion}

Die Algorithmen zur automatischen Anfallsdetektion können entweder auf einen oder auf mehrere EEG-Kanäle angewendet werden. Der Vorteil von Algorithmen, die mehrere oder alle EEG-Kanäle zur Anfallsdetektion verwenden, liegt darin, dass keine A-priori-Festlegungen betreffend der Anfallsursprungszone erforderlich sind. Bei diesen Algorithmen kann die Wahl der Montage von kritischer Bedeutung für die Performance des Algorithmus sein [36].

Generell bestehen Algorithmen zur automatischen Anfallsdetektion aus den folgenden Schritten [37]:

- Prä-Processing, d.h. Artefakterkennung und -bereinigung, Datenfilterung;

- Feature Berechnung/Reduktion/Extraktion, d.h. Berechnung, Reduktion und Extraktion von anfallstypischen Merkmalen aus den EEG-Daten;

- Klassifikation, d. h. Anwendung eines Schwellenwerts oder von modellbasierten Kriterien für die Entscheidung, ob im analysierten EEG-Abschnitt ein Anfall vorliegt oder nicht.

\section{Merke}

Bei Anfallsdetektionssystemen erfolgt zunächst ein Prä-Processing, nachfolgend eine Feature-

Berechnung/Reduktion/Extraktion und schließlich eine Klassifikation, ob im analysierten EEG-Abschnitt ein Anfall vorliegt oder nicht.

Unterschiedliche EEG-Merkmale wurden in zahlreichen Algorithmen zur Anfallsdetektion verwendet:

- morphologische Merkmale in der Zeitdomäne (z. B. Line Length, Halfwaves, Area, Amplitude, Principal Component Analyse etc.),

- biologisch inspirierte Merkmale (z. B. Kreuzkorrelation zwischen den Kanälen, Synchronisation etc.) und

- Frequenzeigenschaften (z. B. Fast-Fourier-Transformation, Wavelet-Transformation etc.).
Die Klassifikationskriterien reichen von einfachen Schwellwertverfahren bis zu komplexen Machine-Learning-Methoden wie Support-Vektor-Maschinen, Random-ForestVerfahren (Entscheidungsbäume) und künstlichen neuronalen Netzwerken [34, 37]. Zuletzt kamen auch DeepLearning-Algorithmen zur Anwendung [38, 39]. Die Algorithmen werden zunächst an einem Trainingsdatensatz trainiert und dann an einem unabhängigen prospektiven Datensatz validiert [30,37].

Zahlreiche Anfallsdetektionssysteme konnten bereits in der klinischen Routine erprobt werden. Die in diesen Studien berichteten Sensitivitäten liegen zwischen 75 und $90 \%$ [24-26].

Cave

Probleme bei der Detektion machen EEG-Anfallsmuster mit kurzer Dauer, niedriger Amplitude, hochgradig umschriebener fokaler Aktivität, hoher Frequenz und ungewöhnlicher nicht-rhythmischer Morphologie sowie Artefakt-verdeckte Muster.

Deshalb sind Anfälle bei extratemporalen Epilepsien schwieriger zu detektieren als Anfälle bei Temporallappenepilepsien [30,34].

Die Spezifität variiert zwischen 0,1-5 falsch-positiven Alarmen (FAR) pro h [24-26]. Niedrige FARs sind für die praktisch-klinische Akzeptanz eines Algorithmus wichtig, und zwar besonders dann, wenn der Algorithmus als Alarmsystem verwendet wird. Hohe FARs würden hier zu einer Verunsicherung des Patienten und zu unnötigen Reaktionen der Angehörigen oder des medizinischen Personals führen. Gründe für falsch-positive Alarme können einerseits physiologische und pathologische EEG-Veränderungen, wie EEG-Muster während des Schlafs sowie frontale intermittierende rhythmische Verlangsamungen (FIRDA) und temporale intermittierende rhythmische Verlangsamungen (TIRDA) sowie andererseits verschiedene, vor allem rhythmische Artefakte sein (z. B. Kauartefakte, Artefakte beim Zähneputzen, rhythmische Bewegungen, Augenbewegungen etc.) $[30,34]$.

Merke

Die Wahl der mit dem Algorithmus analysierten Elektroden (insbesondere die Verwendung basaler temporaler Elektroden) und die gewählten Montagen können die Sensitivität und die Spezifität entscheidend beeinflussen [34].

Um eine stabile Performance zu erreichen, muss ein Algorithmus an einer großen EEG-Datenmenge getestet werden, die auch alle Facetten des interiktalen EEGs über den gesamten Schlaf-Wach-Zyklus, verschiedenste Artefakte und sämtliche physiologische und pathologische EEG-Veränderungen beinhaltet. Der Algorithmus muss deshalb 


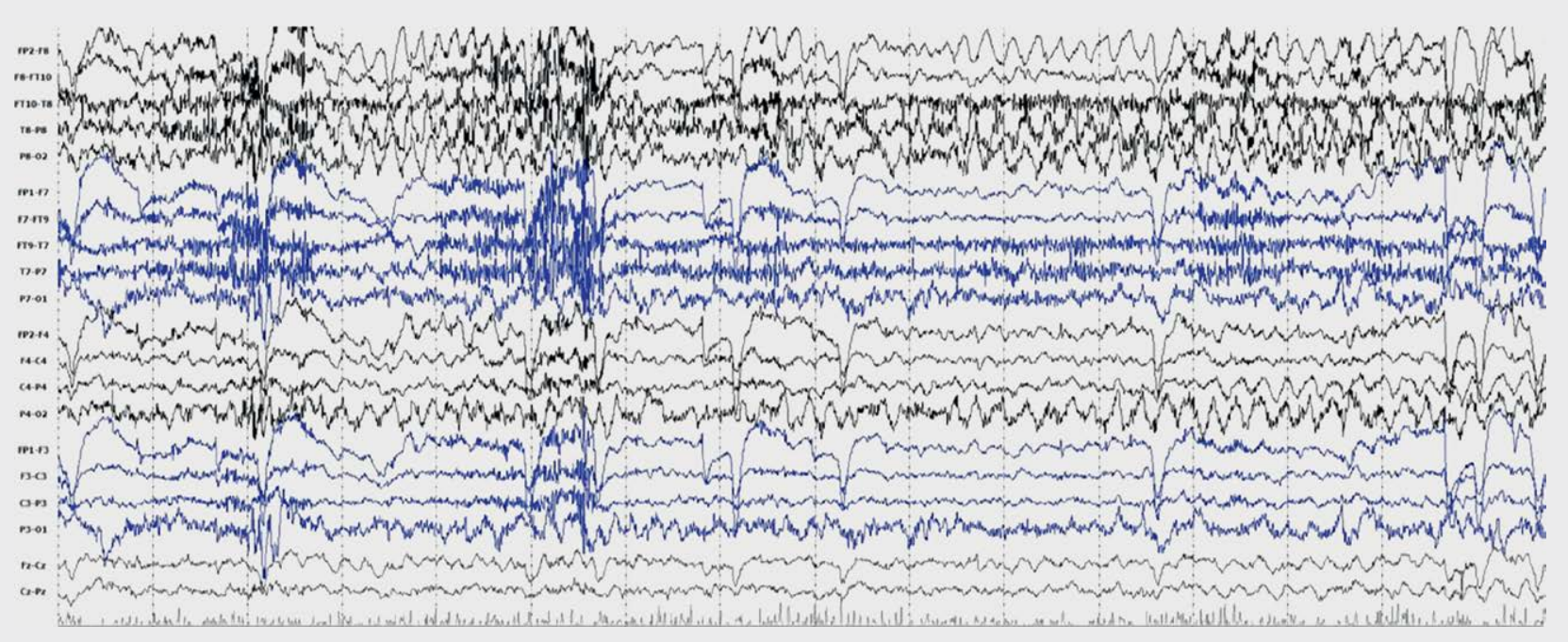

Abb. 3 Iktales EEG - automatische Anfallsdetektion. Anfallsmuster mit rhythmischer Thetatätigkeit rechts temporal (detektiert durch das AnfallsDetektions-Modul EpiScan des EEG-Auswerteprogramms Encevis).

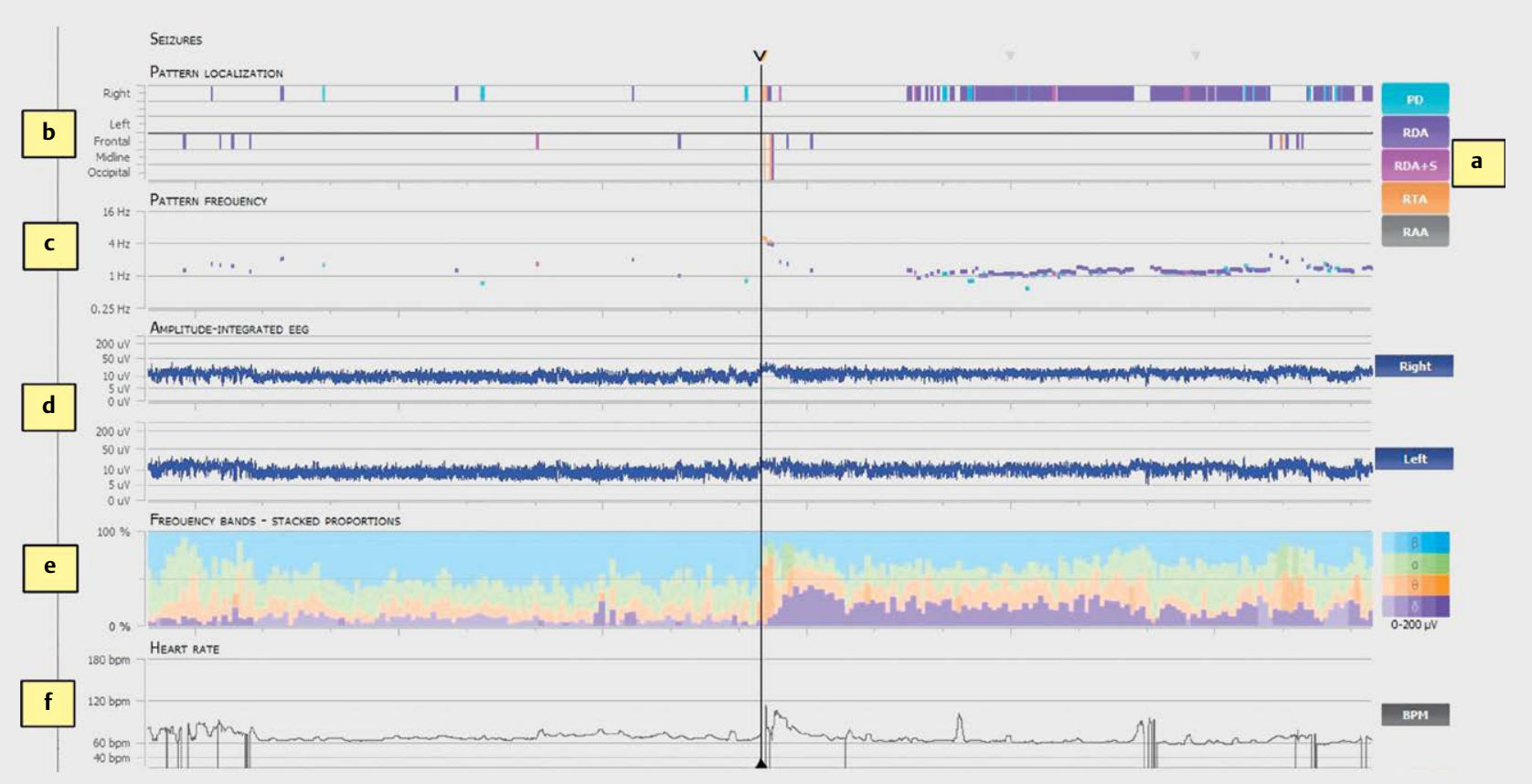

Abb. 4 Automatische Analyse des EEG-Verlaufs mit dem EEG-Trend-Modul NeuroTrend des EEG-Auswerteprogramms Encevis. a. Art des EEGMusters (EEG-Pattern) farbkodiert dargestellt ( $P D=$ periodic discharge, RDA = rhythmic delta activity, RDA + $S=$ rhythmic delta activity with superimposed sharp waves, RTA = rhythmic theta activity, RAA = rhythmic alpha activity). b. Pattern-Localization: Lokalisation des Musters (rechts vs. links sowie frontal, midline, occipital). c. Pattern-Frequency: Frequenz des Musters. d. Amplitude-Integrated EEG: amplituden-integriertes EEG über der rechten und linken Hemisphäre. e. Frequency Bands - Stacked Proportions: Darstellung des relativen Beitrags verschiedener Frequenzbänder zum EEG. f. Heart Rate: Herzfrequenz (BPM = beats per minute).

auch die rasche und robuste Handhabung großer EEG-Datenmengen ermöglichen [30,34].

\section{Merke}

Die Sensitivitäten für automatische

Anfallsdetektionssysteme liegen bei $75-90 \%$ bei $0,1-5$

falsch-positiven Alarmen pro h.
In > Abb. $\mathbf{3}$ ist das Anfallsmuster eines durch das AnfallsDetektions-Modul EpiScan des EEG-Auswerteprogramms Encevis detektierten rechtstemporalen Anfalls dargestellt. In > Abb. $\mathbf{4}$ sind die Ergebnisse der automatischen Analyse des EEG-Verlaufs mit dem EEG-Trend-Modul NeuroTrend des EEG-Auswerteprogramms Encevis zusammengefasst. 


\section{FALLBEISPIEL}

Fallbeispiel Frau E.: Video-EEG-Monitoring - Darstellung des EEGs im zeitlichen Verlauf

In $>$ Abb. $\mathbf{4}$ sind die Ergebnisse der automatischen Analyse des EEG-Verlaufs mit dem EEG-Trend-Modul NeuroTrend des EEG-Auswerteprogramms Encevis zusammengefasst.

Bei unserer Patientin zeigt die computergestützte Auswertung zum Zeitpunkt des detektierten Anfalls (vertikale Linie) eine rhythmische Theta-Tätigkeit (orange-farben; a. in \ Abb. 4) über der rechten Hemisphäre (s. Pattern Localization; b. in >Abb. 4), mit einer Frequenz von etwas mehr als $4 \mathrm{~Hz}$ (s. Pattern Frequency; $\mathbf{c}$. in >Abb. 4). Die Amplitude des EEGs zeigt einen Anstieg über der rechten Hemisphäre (s. Amplitude-Integrated EEG; $\mathbf{d}$. in > Abb. 4), der relative Theta-Frequenzanteil des EEGs nimmt während des Anfalls signifikant auf über $60 \%$ zu (s. Frequency Bands - Stacked Proportions; e. in >Abb. 4). Die Herzfrequenz zeigt einen abrupten Anstieg auf 120 bpm (s. Heat Rate; $\mathbf{f}$. in >Abb. 4).

Zudem können im zeitlichen Verlauf des interiktalen EEGs wiederholte intermittierende rhythmische Delta-Aktivitäten (temporal intermittent rhythmic delta activity - TIRDA, violett) über der rechten Hemisphäre visualisiert werden.

Zusammenfassend ergibt sich somit der Befund einer rechtshirnigen MR-negativen Temporallappenepilepsie.

Neben der Anfallsdetektion mittels EpiScan ermöglicht NeuroTrend somit einen umfassenden Überblick über wesentliche EEG-Parameter im gesamten zeitlichen Verlauf der EEG-Ableitung.

\section{KERNAUSSAGEN}

- Automatische computerunterstützte Methoden zur Detektion von epilepsietypischen Potenzialen (ETPs) und Anfallsmustern ermöglichen eine zeitsparende, objektive, rasch und jederzeit verfügbare quantitative EEG-Befundung.

- Automatische Spike-Detektionsprogramme erkennen ETPs mit einer Sensitivität von 65-99\% bei falsch-positiven Detektionen von 0,09-13,4 pro Minute.

- In rezenten Studien zeigten automatische Spike-Detektionsprogramme eine vergleichbare oder sogar bessere Performance als erfahrene EEG-Befunder.

- Das Anfallserfassungsproblem besteht darin, dass die betroffenen Patienten und ihre Angehörigen die Mehrheit der fokalen, nicht bewusst erlebten Anfälle und der Anfälle aus dem Schlaf nicht bemerken. Automatische Anfallsdetektionssysteme könnten durch eine objektive Anfallsdokumentation zur Lösung dieses Problems beitragen.

- Automatische Anfallsdetektionssysteme könnten zudem zur Verhinderung von anfallsassoziierten Verletzungen, zur SUDEP-Prävention und als Bestandteil innovativer, anfallsgetriggerter, bedarfsgesteuerter Therapien genutzt werden.

- Anwendungen von automatischen Anfallsdetektionssystemen während des Video-EEG-Monitorings bestehen in der Optimierung der Patientensicherheit, der Ökonomisierung der Datenauswertung mit objektiver und reproduzierbarer Quantifizierung von Anfallsmustern und der automatisierten computergestützten iktalen und postikalen Patiententestung.

- Die Sensitivitäten von Anfallsdetektionssystemen liegen bei 75-90\% bei falsch-positiven Alarmraten von 0,1-5 pro h. Anfälle bei extratemporalen Epilepsien sind schwieriger zu detektieren als solche bei Temporallappenepilepsien.

- Machine-Learning-Verfahren und hier insbesondere Deep-Learning-Algorithmen eröffnen eine neue vielversprechende Ära in der automatischen Spike- und Anfallsdetektion.

Interessenkonflikt
Erklärung zu finanziellen Interessen

Forschungsförderung erhalten: nein; Honorar/geldwerten Vorteil für Referententätigkeit erhalten: ja; Bezahlter Berater/ interner Schulungsreferent/Gehaltsempfänger: nein; Patent/ Geschäftsanteile/Aktien (Autor/Partner, Ehepartner, Kinder) an im Bereich der Medizin aktiven Firma: nein. Patent/Geschäftsanteile/Aktien (Autor/Partner, Ehepartner, Kinder) an zu Sponsoren dieser Fortbildung bzw. durch die Fortbildung in ihren Geschäftsinteressen berührten Firma: nein.

Erklärung zu nichtfinanziellen Interessen

Die Autorinnen/Autoren geben an, dass kein Interessenkonflikt besteht.

\section{Autorinnen/Autoren}

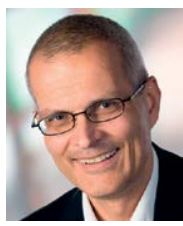

\section{Christoph Baumgartner}

Univ. Prof. Dipl. Ing. Dr. med. univ. Vorstand der Neurologischen Abteilung der Klinik Hietzing; Leiter des Karl Landsteiner Instituts für Klinische Epilepsieforschung und Kognitive Neurologie; Ordinarius für Epileptologie und Klinische Neurophysiologie, Medizinische Fakultät, Sigmund Freud Privatuniversität.

Schwerpunkte: Epilepsie, prächirurgische Epilepsiediagnostik, klinische Neurophysiologie. 


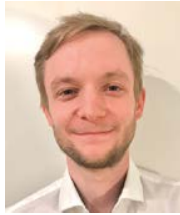

\section{Sebastian Hafner}

Dr. med. univ. 2010-2017 Studium der Humanmedizin an der Medizinischen Universität Wien. Seit 2018 Facharztausbildung Neurologie an der Neurologischen Abteilung der Klinik Hietzing. Schwerpunkte: klinische Neurophysiologie, automatisierte EEG-Analysen, Epilepsie.

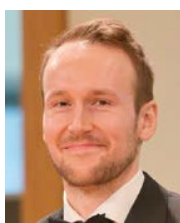

\section{Johannes Peter Koren}

Dr. med. univ. PhD. 2005-2011 Studium der Humanmedizin (Medizinische Universität Wien). Seit 2011 wiss. Mitarbeiter am Karl Landsteiner Institut für klinische Epilepsieforschung. 2018 Abschluss PhD-Studium in Clinical Neuroscience. 2014-2020 Facharztausbildung Neurologie an der Neurologischen Abteilung der Klinik Hietzing. Schwerpunkte: klinische Neurophysiologie, automatisierte EEG-Analysen, Epilepsie, neurologische Intensivmedizin.

\section{Korrespondenzadresse}

Univ. Prof. DI. Dr. med. Christoph Baumgartner Neurologische Abteilung, Klinik Hietzing Karl Landsteiner Institut für Klinische Epilepsieforschung und Kognitive Neurologie

Medizinische Fakultät, Sigmund Freud Privatuniversität Wolkersbergenstraße 1

A-1130 Wien

Österreich

cs.baumgartner@gmail.com

\section{Wissenschaftlich verantwortlich gemäß Zertifizierungsbestimmungen}

Wissenschaftlich verantwortlich gemäß Zertifizierungsbestimmungen für diesen Beitrag ist Prof. Dr. med. Christoph Baumgartner, Wien.

\section{Literatur}

[1] Hartl E, Noachtar S. EEG: Stellenwerte und technische Voraussetzungen der EEG-Untersuchung. Klin Neurophysiol 2018; 49: 48-55

[2] Koren J, Herta J, Baumgartner C. Critical care EEG. Klin Neurophysiol 2019; 50: 60-72

[3] Bagheri E, Dauwels ], Dean BC et al. Interictal epileptiform discharge characteristics underlying expert interrater agreement. Clin Neurophysiol 2017; 128: 1994-2005

[4] Jing J, Herlopian A, Karakis I et al. Interrater reliability of experts in identifying interictal epileptiform discharges in electroencephalograms. JAMA Neurol 2020; 771: 49-57

[5] Webber WRS, Lesser RP. Automated spike detection in EEG. Clin Neurophysiol 2017; 128: 241-242
[6] Noachtar S, Binnie C, Ebersole J et al. Glossar der meistgebrauchten Begriffe in der klinischen Elektroenzephalografie und Vorschläge für die EEG-Befunderstellung. Klin Neurophysiol 2004; 35: 5-21

[7] Baumgartner C, Gallmetzer P, Pirker S et al. EEG-Muster, die mit epilepsietypischer Aktivität verwechselt werden könnn. In: Ebner A, Deuschl G, Hrsg. EEG - Referenzreihe Neurologie. Methoden. 2. Aufl. Stuttgart: Thieme; 2011

[8] Benbadis SR. "Just like EKGs!" Should EEGs undergo a confirmatory interpretation by a clinical neurophysiologist? Neurology 2013; 80: 47-51

[9] Halford J], Arain A, Kalamangalam GP et al. Characteristics of EEG interpreters associated with higher interrater agreement. J Clin Neurophysiol 2017; 34: 168-173

[10] Halford J], Westover MB, LaRoche SM et al. Interictal epileptiform discharge detection in EEG in different practice settings. J Clin Neurophysiol 2018; 35: 375-380

[11] Scheuer ML, Bagic A, Wilson SB. Spike detection: Interreader agreement and a statistical Turing test on a large data set. Clin Neurophysiol 2017; 128: 243-250

[12] Lodder SS, Askamp J, van Putten M]. Inter-ictal spike detection using a database of smart templates. Clin Neurophysiol 2013; 124: 2328-2335

[13] Jing J, Sun H, Kim JA et al. Development of expert-level automated detection of epileptiform discharges during electroencephalogram interpretation. JAMA Neurol 2020; 77: 103-108

[14] Frost JD Jr. Automatic recognition and characterization of epileptiform discharges in the human EEG. J Clin Neurophysiol 1985; 2: 231-249

[15] Wilson SB, Emerson R. Spike detection: a review and comparison of algorithms. Clin Neurophysiol 2002; 113: 1873-1881

[16] Harner R. Automatic EEG spike detection. Clinical EEG and neuroscience 2009: 40: 262-270

[17] Halford J]. Computerized epileptiform transient detection in the scalp electroencephalogram: obstacles to progress and the example of computerized ECG interpretation. Clin Neurophysiol 2009; 120: 1909-1915

[18] Tjepkema-Cloostermans MC, de Carvalho RCV, van Putten M. Deep learning for detection of focal epileptiform discharges from scalp EEG recordings. Clin Neurophysiol 2018; 129: 2191-2196

[19] Gotman J. Automatic recognition of epileptic seizures in the EEG. Electroencephalogr Clin Neurophysiol 1982; 54: 530-540

[20] Smith G, Lee S, van Drongelen W. A glimpse into the future of clinical neurophysiology: Can we use machines to interpret EEG? Clin Neurophysiol 2018; 129: 2186-2187

[21] van Putten M, Olbrich S, Arns M. Predicting sex from brain rhythms with deep learning. Scientific reports 2018; 8: 3069

[22] Hoppe C, Poepel A, Elger CE. Epilepsy: accuracy of patient seizure counts. Arch Neurol 2007; 64: 1595-1599

[23] Elger CE, Mormann F. Seizure prediction and documentation - two important problems. Lancet Neurol 2013; 12: $531-532$

[24] Baumgartner C, Koren JP. Automatic seizure detection in epilepsy. Klin Neurophysiol 2018; 49: 8-20

[25] Baumgartner C, Koren JP. Seizure detection using scalp-EEG. Epilepsia 2018; 59: Suppl 1 14-22 
[26] Baumgartner C, Koren JP, Rothmayer M. Automatic computer-based detection of epileptic seizures. Front Neurol 2018; 9: 639

[27] Ulate-Campos A, Coughlin F, Gainza-Lein M et al. Automated seizure detection systems and their effectiveness for each type of seizure. Seizure 2016; 40: 88-101

[28] Hamandi K, Beniczky S, Diehl B et al. Current practice and recommendations in UK epilepsy monitoring units. Report of a national survey and workshop. Seizure 2017; 50: 92-98

[29] Rosenow F, Bast T, Czech T et al. Revised version of quality guidelines for presurgical epilepsy evaluation and surgical epilepsy therapy issued by the Austrian, German, and Swiss working group on presurgical epilepsy diagnosis and operative epilepsy treatment. Epilepsia 2016; 57: 12151220

[30] Furbass F, Ossenblok P, Hartmann M et al. Prospective multi-center study of an automatic online seizure detection system for epilepsy monitoring units. Clin Neurophysiol 2015; 126: 1124-1131

[31] Beniczky S, Neufeld M, Diehl B et al. Testing patients during seizures: A European consensus procedure developed by a joint taskforce of the ILAE - Commission on European Affairs and the European Epilepsy Monitoring Unit Association. Epilepsia 2016; 57: 1363-1368

[32] Touloumes G, Morse E, Chen WC et al. Human bedside evaluation versus automatic responsiveness testing in epilepsy (ARTiE). Epilepsia 2016; 57: e28-32

[33] Wilson SB, Scheuer ML, Plummer C et al. Seizure detection: correlation of human experts. Clin Neurophysiol 2003; 114: 2156-2164

[34] Hopfengartner R, Kasper BS, Graf W et al. Automatic seizure detection in long-term scalp EEG using an adaptive thresholding technique: a validation study for clinical routine. Clin Neurophysiol 2014; 125: 1346-1352
[35] Meier R, Dittrich H, Schulze-Bonhage A et al. Detecting epileptic seizures in long-term human EEG: a new approach to automatic online and real-time detection and classification of polymorphic seizure patterns. J Clin Neurophysiol 2008; 25: 119-131

[36] Hopfengartner R, Kerling F, Bauer V et al. An efficient, robust and fast method for the offline detection of epileptic seizures in long-term scalp EEG recordings. Clin Neurophysiol 2007; 118: 2332-2343

[37] Ramgopal S, Thome-Souza S, Jackson M et al. Seizure detection, seizure prediction, and closed-loop warning systems in epilepsy. Epilepsy Behav 2014; 37: 291-307

[38] Emami A, Kunii N, Matsuo T et al. Seizure detection by convolutional neural network-based analysis of scalp electroencephalography plot images. Neurolmage Clinical 2019; 22: 101684

[39] Hussein R, Palangi H, Ward RK et al. Optimized deep neural network architecture for robust detection of epileptic seizures using EEG signals. Clin Neurophysiol 2019; 130: 25-37

Bibliografie

DOI https://doi.org/10.1055/a-1169-4254

Klin Neurophysiol 2020; 51: 118-131

(c) Georg Thieme Verlag KG Stuttgart · New York ISSN 1434-0275 


\section{CME-Fragen bei CME.thieme.de}

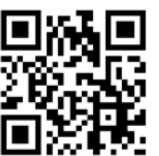

Diese Fortbildungseinheit ist bis zu 12 Monate online für die Teilnahme verfügbar.

Den genauen Einsendeschluss finden Sie beim Modul auf https://eref.thieme.de/CXF1K6R

Sollten Sie Fragen zur Online-Teilnahme haben, finden Sie unter https://cme.thieme.de/hilfe

eine ausführliche Anleitung. Wir wünschen viel Erfolg beim Beantworten der Fragen!

Unter https://eref.thieme.de/CXF1 K6R oder über den QR-Code kommen Sie direkt zum Artikel.

\section{Frage 1}

Welche der folgenden Aussagen trifft nicht zu? Die Erfassung von epilepsietypischen Potenzialen ist wichtig für ...

A die Differenzialdiagnose von anfallsartigen Ereignissen.

B die Prognoseabschätzung nach einem ersten Anfall.

C die Epilepsie-Klassifikation.

D die Lokalisation der irritativen Zone bei fokalen Epilepsien im Rahmen der präoperativen Epilepsiediagnostik.

E die Überprüfung der Therapiegüte bei fokalen Epilepsien.

\section{Frage 2}

Eine der folgenden Aussagen trifft nicht zu. Welche?

A Die visuelle EEG-Befundung ist zeitaufwendig.

B Die visuelle EEG-Befundung erfordert eine spezielle Ausbildung und Erfahrung.

C Fehldiagnosen durch wenig erfahrene EEG-Befunder sind häufig.

D Unter qualifizierten und erfahrenden EEG-Befundern besteht eine perfekte Übereinstimmung bei der Annotation von epilepsietypischen Potenzialen.

E Die visuelle EEG-Beurteilung ermöglicht nur sehr eingeschränkt eine räumliche und zeitliche Quantifizierung von epilepsietypischen Potenzialen.

\section{Frage 3}

Alle folgenden EEG-Transienten können fälschlicherweise als epilepsietypische Potenziale (ETPs) interpretiert werden außer ...
A Wicket-Spikes.
B benigne epileptiforme Transienten des Schlafs.
C anteriore temporale Spikes.
D $14 \& 6 \mathrm{~Hz}$-positive Spikes.
E Phantom-Spike-Wave-Komplexe.

\section{Frage 4}

Automatische Spike-Detektionsprogramme erkennen ETPs mit welcher Sensitivität?
A $10-30 \%$
B $30-40 \%$
C $40-50 \%$
D $50-60 \%$
E $65-99 \%$

\section{Frage 5}

Wodurch ist die Performance von automatischen SpikeDetektionsprogrammen gekennzeichnet?

A Sie haben eine schlechtere Performance als unerfahrene EEG-Befunder.

B Sie haben eine vergleichbare Performance wie unerfahrene EEG-Befunder.

C Sie haben eine schlechtere Performance als erfahrene EEG-Befunder.

D Sie können eine vergleichbare oder sogar bessere Performance als erfahrene EEG-Befunder haben.

E Die Performance kann mit menschlichen EEG-Befunden aus methodischen Gründen nicht verglichen werden.

\section{Frage 6}

Das Anfallserfassungsproblem besteht darin, dass die betroffenen Personen ...

A mehr als 50\% ihrer Anfälle nicht bemerken.

B ihre Anfälle falsch klassifizieren.

$C$ zu viele Anfälle berichten.

D die Anfälle zu falschen Uhrzeiten eintragen.

E ihre Anfälle nicht mit der Medikamentenadhärenz korrelieren.

\section{Frage 7}

Nicht zu den Einsatzmöglichkeiten für automatische computerbasierte Anfallsdetektionssysteme zählt ...
A die objektive Anfallsdokumentation.
B die Anfallsprädiktion.
C der Einsatz als Anfallswarnsystem.
D die SUDEP-Prävention.
E der Einsatz im Rahmen von innovativen bedarfsge- steuerten anfallsgetriggerten Therapien.




\section{CME-Fragen bei CME.thieme.de}

\section{Fortsetzung ...}

\section{Frage 8}

Anwendungsmöglichkeiten für automatische computerbasierte Anfallsdetektionssysteme in der EpilepsieMonitoring-Unit umfassen nicht ...

A die Optimierung der Patientensicherheit.

B die Ökonomisierung der Datenauswertung.

C die Differenzialdiagnose von epileptischen versus nicht-epileptischen psychogenen Anfällen.

D die Quantifizierung von Anfallsmustern.

E die automatisierte computergestützte iktale und postiktale Patiententestung.

\section{Frage 9}

Eines der folgenden Kriterien gehört nicht zu den Anforderungen an ein Anfallsdetektionssystem, das als Warnsystem eingesetzt wird. Welches?

A hohe Sensitivität

B exakte Differenzierung von bewusst und nicht bewusst erlebten Anfällen

C Online-Berechnung

D kurze Detektionslatenz

E niedrige falsch-positive Alarmrate

\section{Frage 10}

Probleme bei der Anfallsdetektion machen welche EEG-

Anfallsmuster?

A EEG-Anfallsmuster mit langer Dauer

B EEG-Anfallsmuster mit hoher Amplitude

C EEG-Anfallsmuster mit niedriger Frequenz

D rhythmische Muster

E Artefakt-verdeckte Muster 